\title{
MS14-P07 | STERIC EFFECT IN TETRACOORDINATED NI(II) COMPLEXES WITH ENAMINOKETONE LIGANDS AND THEIR REACTION PRODUCTS WITH HETEROCYCLIC AMINES
}

Zep, Anna (University of Warsaw, Warsaw, POL)

Tetradentate ONNO coordinating ligands, such a Schiff base type salen/salphen and enaminone type acacen/acacphen, are wide studied ligands in chemistry due to the interest in their applicability. They have been successfully employed as ligands in combination with various metals to give catalyst capable of realizing a variety of synthetic transformations.

In this work two types of $\mathrm{Ni}$ (II) complexes based on acacen type ligands with variation in the N, $\mathrm{N}^{\prime}$ bridge: 1,2-bis(4methylbenzoylvinylamino) ethane $\mathrm{Ni}$ (II) - compound C2 and 1,3-bis(4-methylbenzoylvinylamino) propane Ni (II) compound C3 have been synthesized and characterized by elemental analysis, FT-IR, UV-VIS, ${ }^{1} \mathrm{H}$ NMR and ${ }^{13} \mathrm{C}$ NMR spectral techniques.

In our studies we have observed that due the steric preferences compound C3 exhibit the ability to attach small molecules with $\mathrm{N}$-donors. The structures of compounds C2, C3 and octahedral complexes of C3 with heterocyclic aromatic amines were determined by single crystal X-ray diffraction. Structural changes and the stability of the resulting complexes depending on the substituents in the molecules of $\mathrm{N}$-donors will be discussed. 